NOTICIAS Y COMENTARIOS 


\title{
LOS INCENDIOS FORESTALES. UN RIESGO CANDENTE EN «LA MONTAÑA» ALICANTINA
}

\author{
Enrique Matarredona Coll
}

\section{RESUMEN}

En el presente artículo se analiza el riesgo que los incendios forestales representan para el equilibrio ecológico-paisajístico de ciertos espacios de La Montaña alicantina que, en el período 1981-95, ha sido afectada en un total de $31.970 \mathrm{Ha}$. El trabajo se basa en el análisis de los datos extraídos de los Informes sobre Incendios Forestales de la Unidad Forestal de los Servicios Territoriales de la Consejería de Agricultura y Pesca de Alicante, sobre la fecha de los siniestros, la hora de comienzo y extinción, la superficie geográfica quemada, el tipo de vegetación afectada, así como la causalidad del evento; todo ello se ha completado con una observación de campo en sectores con condiciones eco-topográficas distintas, asolados por fuegos en años diferentes, al objeto de poder comprobar in situ las consecuencias de tipo erosivo y el grado de regeneración de la cubierta vegetal.

Palabras clave: Incendios forestales, desertificación, regeneración, medio ambiente, repoblaciones forestales, política forestal, erosión.

\begin{abstract}
It is analyzed in the present article, the risks that forest fires represent for the ecologic balance of certain spaces of La Montaña, in Alicante, where during the period 1981-1995 has been affected in a total of 31,970 Hectares. This work is based on the analysis on the date of the studies about forest fires, investigated in the Unidad Forestal de los Servicios Territoriales (Consejería de Agricultura y Pesca de Alicante), about the hour they beginning and when they were extincted, the way the surface and the vegetation were affected, as well as the causality of the event. Apart from that, it has been carried out a fieldwork in sectors with different environmental conditions, all of them affected by fires in different years, to the object of checking 'in situ' the erosive consequences as well as the stage of regeneration in the vegetable cover.
\end{abstract}

Key words: forest fires, desertification, forest repopulations regeneration, environment, forest policy, erosion. 
Los incendios forestales suponen un fenómeno de combustión violenta de los compuestos químicos contenidos en las formaciones vegetales, con liberación de gran cantidad de energía. Aunque secuencialmente afectan a La Montaña alicantina, sobre todo en verano, no son un fenómeno exclusivo de nuestra época ni de nuestro ámbito geográfico, sino que deben entenderse como un proceso más extenso, si bien se muestra más amenazante en áreas del ámbito mediterráneo, en donde las condiciones medioambientales implican fuertes sequías estivales y generan una vegetación esclerófila de alta combustibilidad, y en las que el fuego - factor ecológico en el desarrollo y evolución de la vegetación forma parte de la dinámica ecológica, con unas especies vegetales capaces de rebrotar tras los incendios (encina, coscoja, madroño), o de protagonizar una intensa diseminación (pino); si bien, es evidente que esta capacidad de supervivencia guarda relación con un ciclo de incendios natural, y no con la dinámica de fuegos de los últimos años — factor de degradación de muchas hectáreas de monte mediterráneo- que está asolando las tierras alicantinas (Figura 1).

\section{Incendios forestales (1981-95). La Montaña}

$\mathrm{N}^{\circ}$

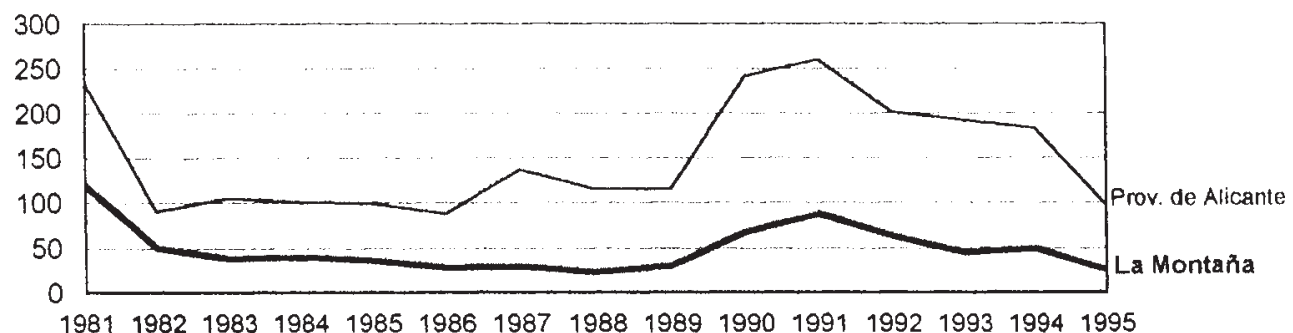

Matamedona

Figura 1. Evolución del número de fuegos en el período 1981-95. La tenue regresión en el número de siniestros en La Montaña en el período 1981-89, se vio incrementada de forma preocupante en el bienio 1990-91, para volver en el último año a aquellas cotas.

En efecto, en el último lustro se han alcanzado cotas alarmantes en lo que se refiere no sólo a hectáreas quemadas -más de 41.800 en los 2.261 incendios declarados a nivel provincial en el período 1981-95-, y enormes pérdidas económicas — se estima una valoración aproximada de 2.000 ptas./Ha. de matorral quemado y de 200.000 ptas./Ha. de pinada incendiada - , sino también en lo referente a degradación paisajística e incremento de los riesgos de erosión que, dada su magnitud, cuestionan en gran medida la capacidad de supervivencia y regeneración de muchas especies.

En el presente artículo analizamos el riesgo que los incendios forestales representan para el equilibrio ecológico-paisajístico de ciertos espacios de La Montaña alicantina. Para alcanzar ese objetivo, hemos basado el trabajo en el análisis de los Informes sobre Incendios Forestales de la Unidad Forestal de los Servicios Territoriales de la Consejería de Agricultura y Pesca de Alicante, que nos han facilitado datos sobre la fecha de los siniestros, la hora de comienzo y extinción, el área geográfica afectada, las hectáreas quemadas, el tipo de superficie siniestrada (arbolada o matorral), así como la causalidad del evento; por otro lado, se ha manejado la información aparecida en la prensa alicantina (diario Información) relativa a los principales incendios forestales acontecidos en el área de estudio en el período comprendido entre 1981 y 1995 (Figuras 2 y 3). Asimismo, hemos 
realizado una labor de observación de campo en parcelas o sectores con condiciones ecotopográficas distintas, afectados por fuegos en años diferentes, al objeto de poder comprobar in situ el efecto de posibles procesos de erosión en zonas quemadas o, incluso, grados de mayor o menor regeneración de la cubierta vegetal, que nos ha permitido enunciar algún tipo de consideraciones.

Incendios forestales. La Montaña

Siniestros más destacados en el período 1981-95

\begin{tabular}{|c|c|c|c|c|}
\hline Fecha & $\begin{array}{c}\text { Monte } \\
\text { Partida }\end{array}$ & $\begin{array}{l}\text { uperficie } \\
\text { Idiada (Ha.) }\end{array}$ & Término Municipal & Causas \\
\hline \multicolumn{5}{|l|}{1981} \\
\hline 3 -II & Tagarina y Aitana & 480 & Sella y Confrides & Intencionado \\
\hline 11-VIII & Solana Bichauca & 280 & Tárbena y Bolulla & Id. \\
\hline $2-X$ & Loma de la Arena & 720 & Planes y Beniarrés & Id. \\
\hline $\begin{array}{c}18-X I I \\
1982\end{array}$ & Solana de Bichauca & 297 & Tárbena y Bolulla & Id. \\
\hline $\begin{array}{c}11-\text { VIII } \\
\mathbf{1 9 8 3}\end{array}$ & Seguró y Villaseca & 275 & Sella, Penáguila y Alcolecha & Desconocidas \\
\hline $11-\mathrm{VI}$ & Diago & 350 & Penáguila & Rayo \\
\hline 22-VII & Plá del Sirer & 225 & Penáguila y Alcolecha & Intencionado \\
\hline 16-VIII & Umbría, Llano Alcalá & 249 & Vall de Gallinera & Id. \\
\hline $\begin{array}{c}25-I X \\
1984\end{array}$ & Escuder, REgall & 450 & Penáguila & Id. \\
\hline 10-VIII & Surcacho, Meco & 175 & Penáguila & Intencionado \\
\hline \multirow{2}{*}{\multicolumn{5}{|c|}{1986}} \\
\hline & & & & \\
\hline 23-VI & Foyetas, Umbría & 420 & Alcalá, Gallinera, Ebo & Desconocidas \\
\hline $6-$ VII & Racó de Lariola & 500 & V. de Laguart, Castells & Intencionado \\
\hline \multicolumn{5}{|l|}{1990} \\
\hline 24-VII & Umbría, Llano de Alcalá & 1.100 & V. de Gallinera, V. de Ebo & Intencionado \\
\hline 24-VII & Monte Solana & 450 & V. de Gallinera & Id. \\
\hline 25-VII & Salem (Valencia) & 800 & Beniarrés, Lorcha & Proc. Valencia \\
\hline \multicolumn{4}{|l|}{1991} & Rayo \\
\hline 30-VII & Safor. La Solana & 1.055 & Lorcha, V. de Gallinera & Proc. Valencia \\
\hline 1-VIII & Benicadell & 165 & Beniarrés, Gallanes, Muro & Id. \\
\hline $1-\mathrm{VIII}$ & Cobeta de Llosco & 400 & Vall de Ebo & Intencionado \\
\hline 18-VIII & Les Murles & 1.200 & Tárbena y otros & Id. \\
\hline 19-VIII & La Rabosa & 500 & Tárbena, Bolulla y otro & Id. \\
\hline 26-VIII & Xorquet, Negre & 950 & Castells, Bolulla y otro & Id. \\
\hline $\begin{array}{c}\text { 27-VIII } \\
1992\end{array}$ & Barranco Ares, Xarquer & 450 & Confrides, Benasua & Id. \\
\hline 29-VIII & Bichauca, Jubeas. & 1.760 & Castells, Laguart, Tárbena & Intencionado \\
\hline $5-X$ & Umbría o Llano de A. & 400 & Lorcha, V. Gallinera & Id. \\
\hline $5-X$ & La Umbría & 300 & Benimantell & Id. \\
\hline \multicolumn{5}{|l|}{1993} \\
\hline $10-\mathrm{VII}$ & Barranco Cocons & 575 & Lorcha, V. Gallinera & Negligencia \\
\hline $\begin{array}{l}3 \text {-IX } \\
1994\end{array}$ & Fuente Nueva, Tossal & 260 & Castells, Famorca, ... & Intencionado \\
\hline 4-VII & Tossal Blanc, Serreta & 1.085 & Muro, Agre, Gayanes & Proc. Valencia \\
\hline 9-VIII & Patró, Benisili, Xarpola & 183 & Alcalá, Planes, Gallinera & Intencionado \\
\hline $\begin{array}{c}21-\text { VIII } \\
1995\end{array}$ & Gormaig, Serelles, Mola & 380 & Cocentaina, ... & Id. \\
\hline 13-VII & Tagarina, Aitana & 502 & Sella, Confrides & Desconocidas \\
\hline 12-VIII & Pas del Pont & 190 & Sella, Confrides, Alcolecha & Intencionado \\
\hline
\end{tabular}

FiguRA 2. Principales incendios forestales de La Montaña en el período 1981-95, según informes de la Unidad Forestal de Servicios Territoriales de la Consejería de Agricultura y Pesca de Alicante. 


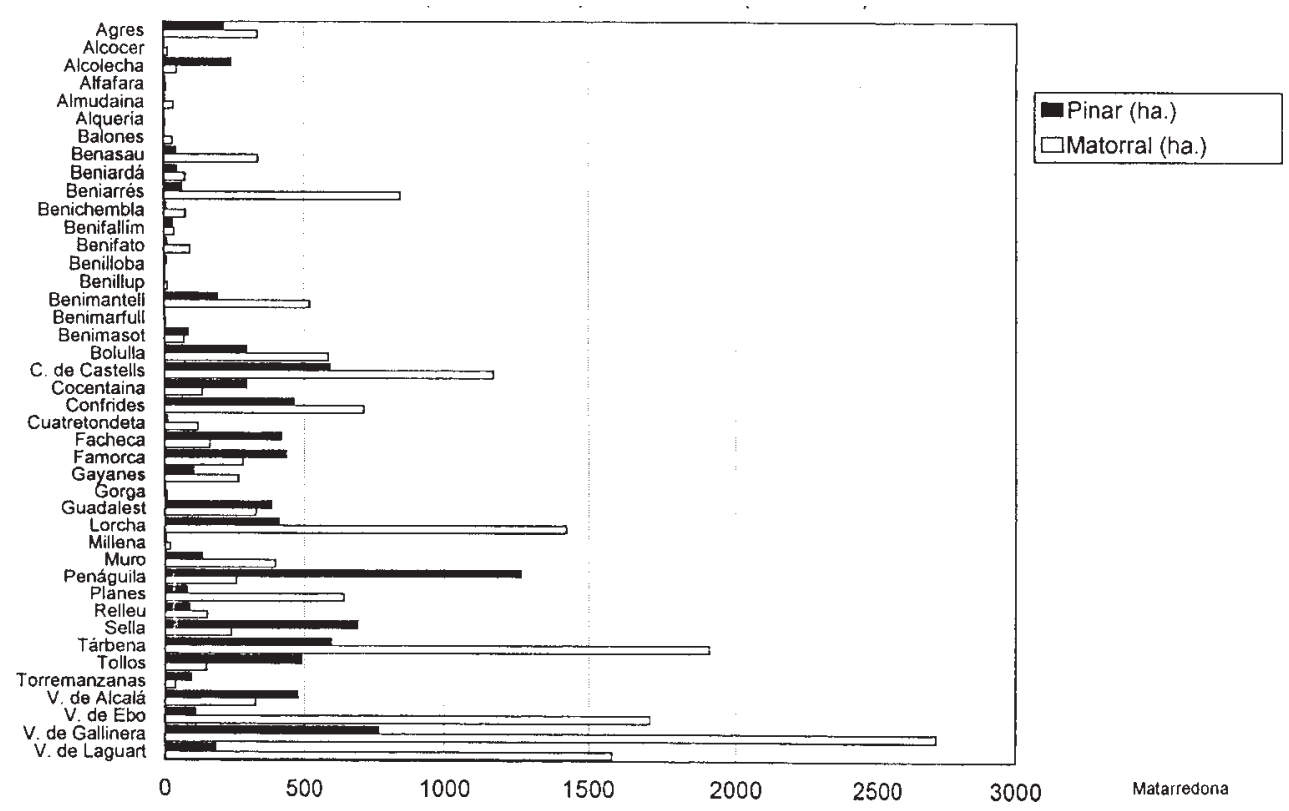

FIGURA 3. Superficie municipal incendiada en el período 1981-95. La mayor área afectada corresponde a la superficie de matorral de los municipios localizados al NE de la comarca, mientras que, el municipio más afectado en lo que se refiere a pinar fue Penáguila.

\section{Condiciones naturales determinantes de los incendios forestales}

Existen una serie de factores que, a nivel general, condicionan el desarrollo de los incendios forestales (Figura 4). Unos son biológicos y dependen de la composición, estructura y disposición de la masa vegetal que, en el caso que estamos analizando, se ajusta al xeromorfismo y pirofitismo de la vegetación mediterránea, con unas especies adaptadas a la sequedad ambiental (presencia de espinas, disminución del tamaño de las hojas, alto contenido en resinas, escasez de agua) o bien predispuestas a la reproducción estimulada por el fuego (rebrotes rápidos postincendio, en el caso de la coscoja; positiva germinación de brezos y romeros; o, incluso, la rápida diseminación de las semillas en los pinares). Estas propiedades xerofíticas y pirofíticas de las especies mediterráneas, dominantes en La Montaña, facilitan la aparición y propagación de los incendios forestales.

Por otra parte, es fundamental la influencia de parámetros climáticos, sobre todo la presencia, intensidad y dirección del viento que, en casos extremos (ponents), supone un aporte constante y violento de oxígeno que actúa de comburante en el proceso. Y todo ello sin olvidar la función del grado de humedad atmosférica y de la propia vegetación - elemento combustible del proceso-, teniendo en cuenta que parte de la energía desarrollada - calor del propio fuego - es empleada en evaporar el agua ambiental y los tejidos vegetales; de ahí que la lluvia en los meses previos al estío pueda considerarse un factor acelerador del riesgo por la proliferación del matorral que puede provocar, del 
mismo modo que las olas de calor también pueden concebirse como agentes detonantes del proceso, sobre todo cuando van acompañadas de desecantes vientos de poniente.

Finalmente, los factores topográficos determinan el frente de avance del fuego y condicionan su velocidad de propagación, teniendo en cuenta que estos eventos progresan ladera arriba en el sentido del viento reinante; de ahí que las zonas abruptas de La Montaña se muestren más proclives a los incendios que los espacios llanos.

De todo ello, y de cara a la prevención de incendios forestales, podemos deducir que sólo los factores biológicos son susceptibles de ser modificados por el hombre, de ahí que, aproximando la vegetación al «clímax», la incidencia del fuego será menor —repoblaciones de Quercus, por ejemplo-, mientras que políticas repobladoras con especies pirofíticas (pinos) o en lugares topográfica y climáticamente inadecuados, incrementarán el riesgo.

\section{CONDICIONES NATURALES DETERMINANTES DE LOS INCENDIOS FORESTALES}

FACTORES BIOLÓGICOS
Dependen de la composición, estructura y

disposición de la masa vegetal.

VEGETACIÓN MEDITERRÁNEA

Xeromorfismo ......Adaptación sequedad ambiental

Pirofitismo ......Reproducción estimulada por fuego

Respuesta vegetativa rápida e inmediata

FACILITAN APARICIÓN Y

PROPAGACIÓN INCENDIOS

Presencia, intensidad y dirección del viento

PONIENTE

Grado de humedad atmosférica y de la vegetación

SEQUEDAD ESTIVAL

Lluvias primaverales: factor de proliferación del matorral

MECHA Y ELEMENTO COMBUSTIBLE DEL MONTE

Determinan frente de avance del fuego

TOPOGRÁFICOS
Ladera arriba en sentido del viento reinante

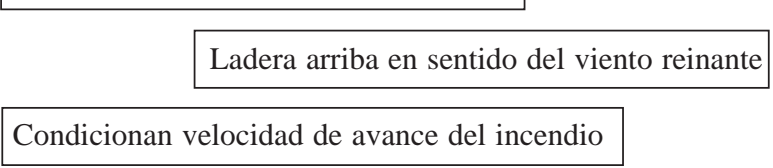

FigURA 4 


\section{Factores que incrementan el riesgo de incendios en La Montaña}

La Montaña alicantina, muy erosionada y explotada, y vulnerable a los incendios forestales, ha estado sometida a una degradación continua. En efecto, integrada de lleno en unos condicionamientos medioambientales de carácter mediterráneo, sus áreas boscosas han tenido graves problemas de regeneración, tanto por estos parámetros climáticos como por los aprovechamientos y actividades a que ha estado sometida (Figura 5).

En efecto, la montaña como recurso, el aprovechamiento del bosque y de la montaña - carrasca y pino, sobre todo- ha sido tradicional en este ámbito alicantino, sin olvidar la explotación del matorral - esparto, barrilla, aliaga, coscoja - que ha tenido una notoria utilidad en el conjunto de la economía tradicional de La Montaña. La mayor parte del terreno montañoso en la zona estudiada ha sido objeto de aprovechamiento por parte del hombre, de ahí que este espacio alicantino lleve la huella indeleble de la acción humana; así el carácter de ámbito humanizado se manifiesta por una organización espacial que se ha ido configurando en el tiempo, sin que se hayan producido modificaciones sustanciales hasta hace unos 30 ó 40 años, momento en el que esa organización ancestral parece entrar en crisis.

\section{Incendios forestales}

La Montaña

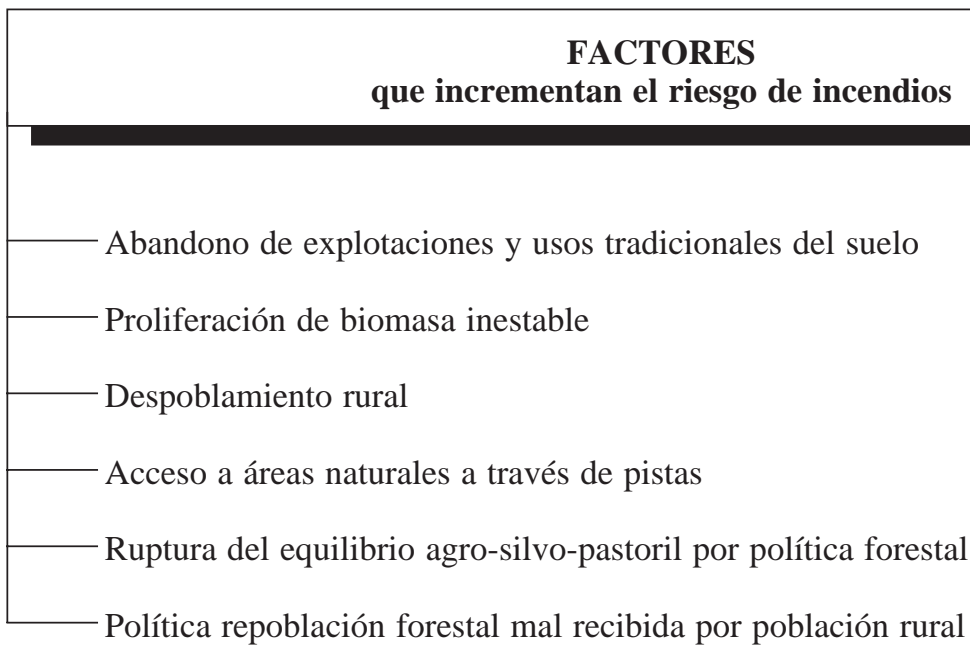

Matarredona

FIGURA 5

Aparte del aprovechamiento tradicional de la madera, en forma de troncos más o menos gruesos, según la finalidad, era significativa la utilización de todo tipo de leña o menuda; el tratamiento, las técnicas empleadas para su obtención, las regulaciones de la actividad (ALTAMIRA, 1985) y el utillaje a emplear variaban según las zonas y el destino final. Así, en La Marina se consumía, preferentemente, madera de Aitana y del Cabeçó d'Or; mien- 
tras que los carpinteros de ribera de la Vila usaban la procedente de Aigües de Busot. Asimismo, la zona posibilitaba el aprovechamiento de otros productos, distintos a pinos o carrascas; se trataba de engilages y ramas resultantes de la poda y limpieza de las zonas arboladas, que daban actividad a gran número de leñadores. El producto recogido se solía dedicar al consumo doméstico, o bien para hornos de pan, de yeso, de cal, o de cerámica.

Por otro lado, la tala ofrecía otros fines como eran los relacionados con el carboneo, actividad bastante especializada del trabajo del monte; usualmente, los carboneros trabajaban en el propio término municipal, casi siempre en tierras de uso comunal. Los tratos se hacían de forma similar a los de los leñadores, debido a que el trabajo previo de la carbonera era la obtención de leña, bien fruto de la limpieza del monte o de la poda de los árboles. Al principio, las carboneras se hacían en época estival, cuando la leña estaba recogida, y también por ser una época menos expuesta a fuertes vientos que podían resultar peligrosos para las carboneras; más recientemente, y hasta la desaparición de esta actividad, se impuso hacerlas todo el año, excepto en los períodos de mal tiempo, como un medio para obtener más beneficios económicos.

El abandono de explotaciones y de los usos tradicionales del suelo, en estos espacios montanos y, con ello el de estas actividades — recolección de leña, limpieza del monte, obtención de carbón vegetal- que otrora supusieron un acusado beneficio para estos espacios, si se realizaban de un modo ordenado, ha contribuido, indudablemente, a incrementar el índice de combustibilidad de las masas forestales y con ello a potenciar el riesgo de incendios; masas forestales carentes, hoy en día, de labores de limpieza lógicas, que contribuyan a la desaparición de residuos vegetales muertos acumulados en la tierra, constitutivos de una biomasa inestable que, evidentemente, acrecienta esos riesgos de fuego.

La evolución demográfica de La Montaña y las nuevas estructuras económicas son, en última instancia, responsables de las transformaciones experimentadas en los últimos años. El proceso de cambio demográfico se ha manifestado en el descenso neto de la población y la brusca transformación estructural, con tasas de envejecimiento que desbordan lo admisible para garantizar la evolución y estabilidad de los efectivos, con las consiguientes repercusiones en el mantenimiento de la tradicional dedicación agrícola y forestal de la zona (MATARREDONA, 1996). Es decir, el proceso de despoblación puede considerarse como factor del desmoronamiento de la organización social y del aprovechamiento del monte.

En efecto, el proceso de despoblación de los últimos cuarenta años ha conducido a una destrucción de la organización social y de las explotaciones agrarias (MATARREDONA, 1996), que se ha traducido en una reducción del espacio agrícola — puesto de manifiesto en el proceso de abandono de bancales-, en una disminución de la variedad de cultivos propia del sistema tradicional, y en el abandono de prácticas agro-forestales ancestrales que contribuían a conservar el suelo y a limpiar los bosques de residuos vegetales (MUÑOZ, 1986).

El descenso de la presión humana sobre el territorio montano está teniendo consecuencias inmediatas en la dinámica del paisaje. Las antiguas laderas aterrazadas, hoy en día abandonadas, se han visto sometidas a un proceso de invasión por parte de las herbáceas y el matorral, y a una nueva dinámica geomorfológica. Sobre los suelos calizos, propios de estos campos abandonados, es frecuente la presencia de un matorral aclarado, a veces raquítico, constituido por romero, tomillo y aliaga, entre otros, que, en casos de mayor densidad constituye un riesgo elevado para la propagación del fuego —el 70\% de la superficie afectada en La Montaña corresponde a matorral- (Figura 6).

Otra reflexión general es el importante deterioro que experimentan estos bancales abandonados, dotados normalmente de gran productividad; y es que, cuanto mayor es la intervención antrópica en una ladera más díficil es que ésta pueda permanecer en equilibrio en ausencia de dicha actuación. Es decir, la presencia humana es imprescindible para conservar 

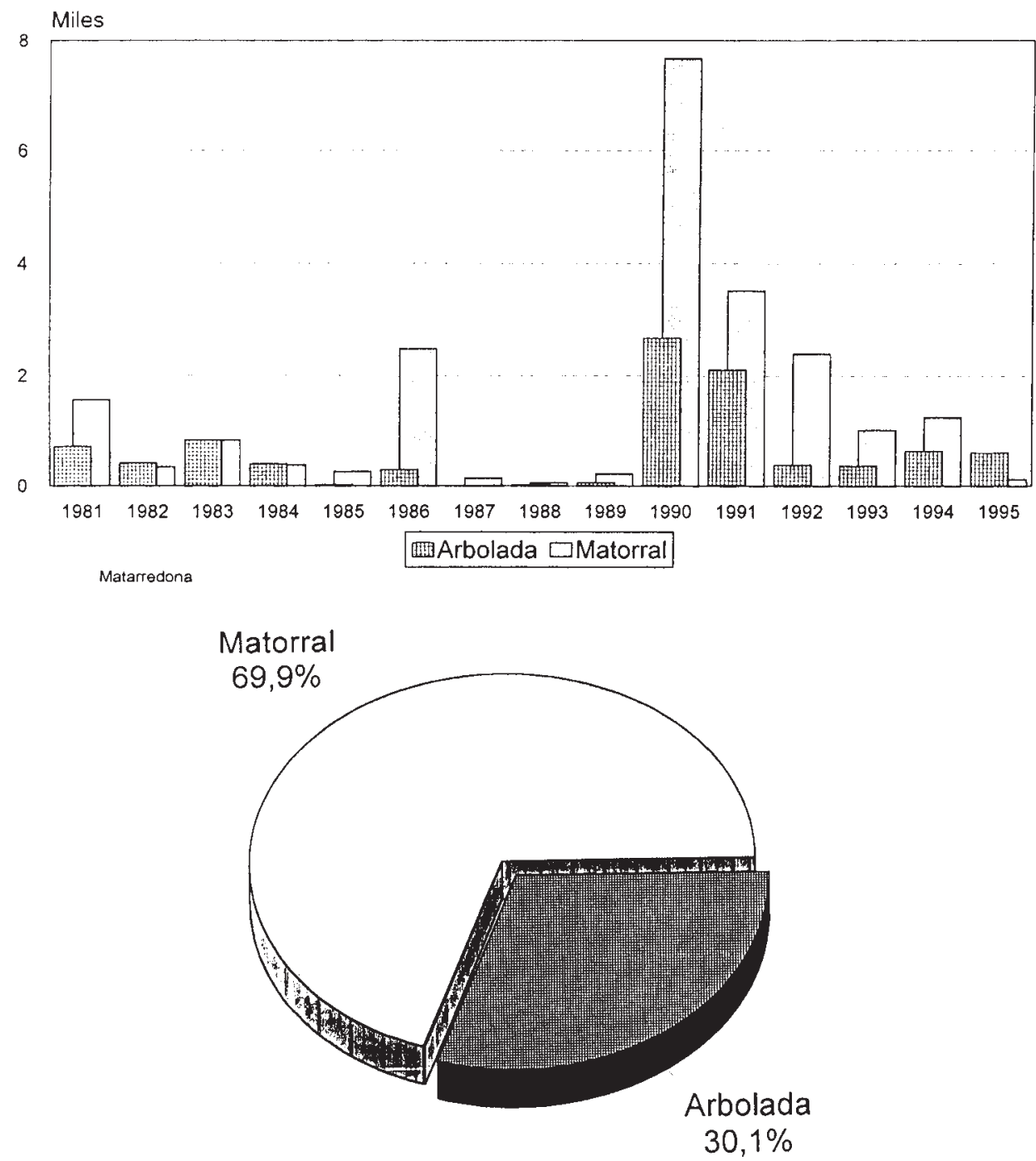

FIGURA 6. Superficie afectada por incendios forestales (1981-1995). Se observa un predominio del área incendiada de matorral (70\%) respecto al dominio de pinar.

aquello que su actuación ha puesto en situación inestable. El problema actual no es que existan los bancales, sino que no se cuiden; un sistema que frena la escorrentía y favorece la infiltración, beneficia al régimen hídrico de las vertientes y reduce las posibilidades de arrastre de los materiales, mientras que, con el abandono, genera la proliferación de una biomasa combustible que acelera la extensión del incendio al no actuar como cortafuego. 


\section{Causas de los Incendios forestales \\ La Montaña (1981-1995)}

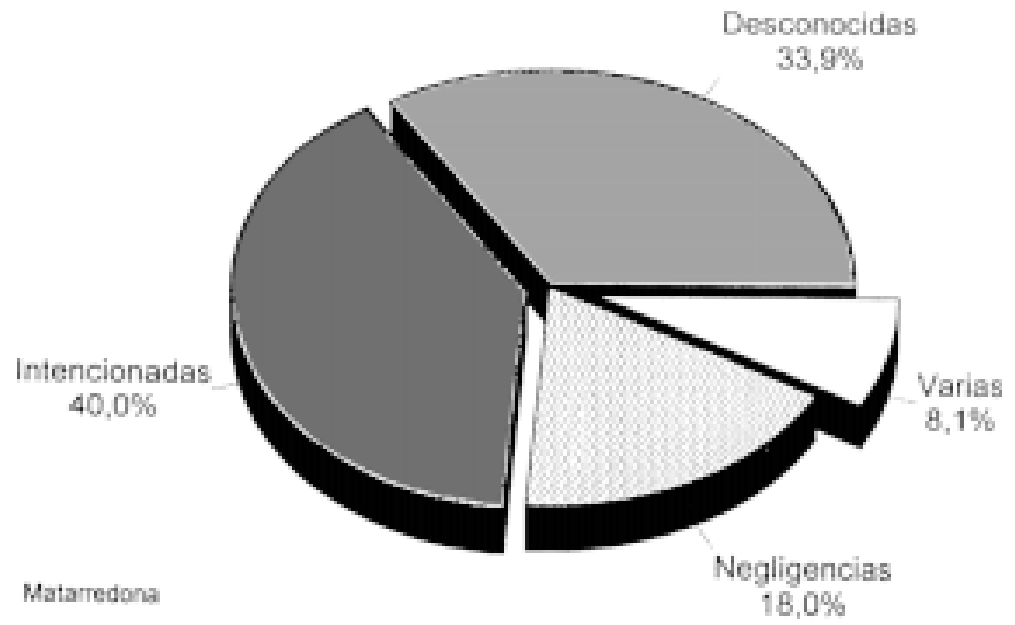

FIGURA 7. La causalidad de los incendios forestales se vincula mayoritariamente a motivaciones intencionadas o causas desconocidas.

Aparte de todo ello, hemos de considerar que en la actualidad ha aumentado el riesgo de incendios debido tanto al abandono de los tradicionales métodos de aprovechamiento forestal, que hemos indicado, como a la pérdida de valor económico de los disfrutes del monte (MONTIEL, 1990) y al proceso de despoblación rural a que se ha visto sometida La Montaña.

Asimismo, contribuye al incremento del índice de riesgos de incendios, la estructura de la propiedad y de uso del suelo forestal. En efecto, el estudio del fenómeno desde la perspectiva de la propiedad de los montes — más del 75\% del bosque quemado en la provincia de Alicante es propiedad privada - nos permite entender la naturaleza de las causas que los provocan, los objetivos que persigue el incendiario y el significado que para la conservación de la masa forestal posee la titularidad de los predios. En este sentido, conviene señalar la trascendencia que desde un primer momento tuvieron las negligencias y la intencionalidad en la provocación de estos procesos. Así, durante los siglos XVIII y XIX es frecuente la información de los archivos relativa a la intervención de los ganaderos para favorecer el crecimiento de los pastos, o la de los agricultores para ampliar las parcelas de cultivo. En la actualidad, los datos manejados indican que la causalidad de los incendios forestales en La Montaña se vincula en un $40 \%$ a causas intencionadas, y en un $33,9 \%$ a motivos desconocidos; mientras que el $18 \%$ corresponde a negligencias relacionadas con quema de márgenes, o de broza, quema de vertederos, descuidos de cazadores o excursionistas, etc. (Figura 7). En este sentido resulta, como mínimo, sorprendente que buen número de fuegos en La Montaña, no el máximo, se inicien en horas nocturnas cuando la presencia humana es menor y los parámetros climáticos no son los de extremado riesgo; con todo, la mayor frecuencia se da en las horas centrales del día (desde las 12 a las 17 horas), coincidiendo con temperaturas ambientales más altas, o incluso, en el caso de negligencias, con una mayor presencia humana (Figura 8). 


\section{La Montaña}

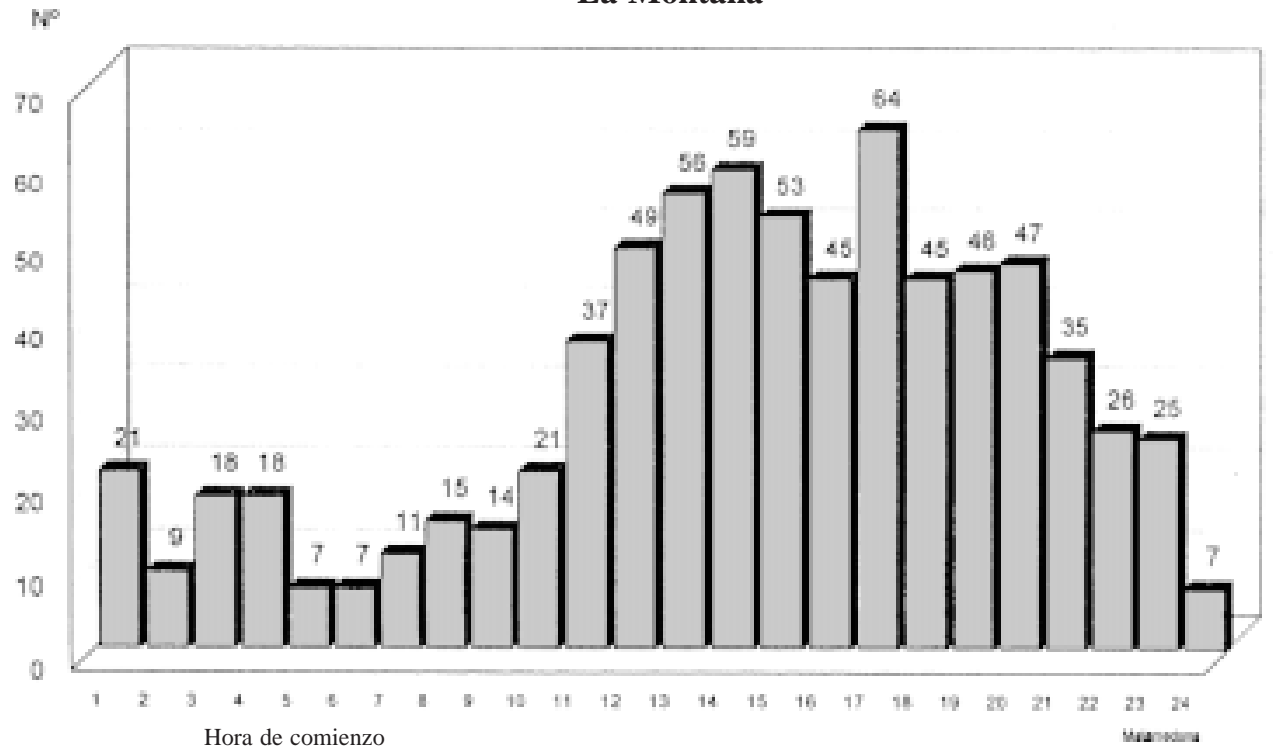

FiguRA 8. Pese a la mayor frecuencia de inicio de los incendios en las horas centrales del día, resulta sorprendente la importancia de las horas nocturanas que, teóricamente, deberían presentar menor relevancia.

Si bien resulta difícil calibrar la intencionalidad, existen, para los técnicos en extinción y prevención de incendios, indicios delatores de tal actitud: focos en dos o tres puntos al mismo tiempo, colocación de piedras en forma de fogata, o incluso, el hallazgo de montones de paja o matorral usados como foco. Aunque en este punto debemos indicar que, según ICONA, la mayoría de los incendios que afectan a superficie de matorral, son provocados para facilitar al ganado trashumante, que llega en otoño procedente de Andalucía y La Mancha, el pasto necesario, toda vez que con el fuego desaparece el matorral excesivamente alto para el ganado, que se nutre de los jóvenes rebrotes que aparecen al poco de producirse el siniestro (a las 8 ó 10 semanas, según las zonas).

Es evidente que el mayor número de incendios se produce en montes de propiedad particular, lo cual es una prueba más del carácter intencionado de los mismos y viene a sumarse al capítulo de las nefastas consecuencias de la privatización de los montes públicos. Por otro lado, la existencia en los montes de utilidad pública de La Montaña, de fincas de propiedad particular (enclavados), que durante el siglo pasado estuvieron dedicadas al cultivo agrícola y que en la actualidad han quedado abandonadas a la colonización del matorral, han podido suponer la pérdida de su función de cortafuegos (GENERALITAT VALENCIANA, 1986). Por otro lado, la concentración en los núcleos urbanos de la población rural, en otro tiempo dispersa, ha privado al monte de unos vigilantes espontáneos.

El incremento de la presencia humana en los fines de semana y el fenómeno de las actividades recreativas en el monte, viene a aunarse, en algunos lugares, con la escalada del proceso de especulación del suelo forestal con el fin de extender las zonas urbanas y 
turísticas (LIEBANA-RIBARROCHA, 1981), al tiempo que la facilidad de acceso a las zonas forestales y la moda del $4 \times 4$ - falso turismo de aventura - ha contribuido a acrecentar el riesgo, quedando así desprotegidas áreas que, hasta hace poco, se encontraban salvaguardadas por su aislamiento (COLMENAR, 1985).

Finalmente hay que citar la ruptura del equilibrio agro-silvo-pastoril, con una política forestal y de repoblaciones que han generado un mal ambiente entre la población rural, en otra época «verdaderos guardianes» de estos espacios montanos (NAVARRO-XANDRI, 1995).

\section{Repercusiones paisajísticas y en el equilibrio ecológico}

Los efectos producidos por los incendios forestales son, a todas luces, negativos para la conservación de los montes y para el mantenimiento del equilibrio ecológico (MONTIEL, 1990). Las consecuencias inmediatas que generan son: la destrucción de la cubierta vegetal, sobre todo en incendios de pinar que producen gran cantidad de calor que dificulta la regeneración; la desprotección de los suelos y la disminución de su porosidad y, por tanto, de la capacidad de infiltración y retención del agua; la reducción de la disponibilidad hídrica, la alteración de las condiciones edáficas y la distorsión del ciclo hidrológico. A todo ello vienen a sumarse el aumento del albedo de la superficie del monte, a causa de las cenizas y, en consecuencia, el aumento de absorción de calor y de potencialidad de evaporación (Figura 9).

\section{Incendios forestales \\ La Montaña}

\section{REPERCUSIONES}

Destrucción de la cubierta vegetal, sobre todo en incendios de pinar que producen gran cantidad de calor dificultando la regeneración

Desprotección de los suelos e incremento de la erosión con pérdida de suelo fértil

Destrucción o alteración de la materia orgánica del suelo y disminución de su fertilidad

Multiplicación de insectos y hongos xilófagos — amenaza potencial para la masa vegetal vecina-, en troncos calcinados y tocones muertos

Cambio de las condiciones microclimáticas, con variaciones térmicas acusadas, menor infiltración y mayor evaporación

Reducción sensible de la población animal, especialmente la microfauna: (gasterópodos, formícidos y arácnidos) así como roedores y reptiles

Incremento de la virulencia de las avenidas fluviales por modificación de la infiltración del agua por el suelo

Pérdida en valores ambientales-Pérdida de «paisaje» Matarredona

FIGURA 9 


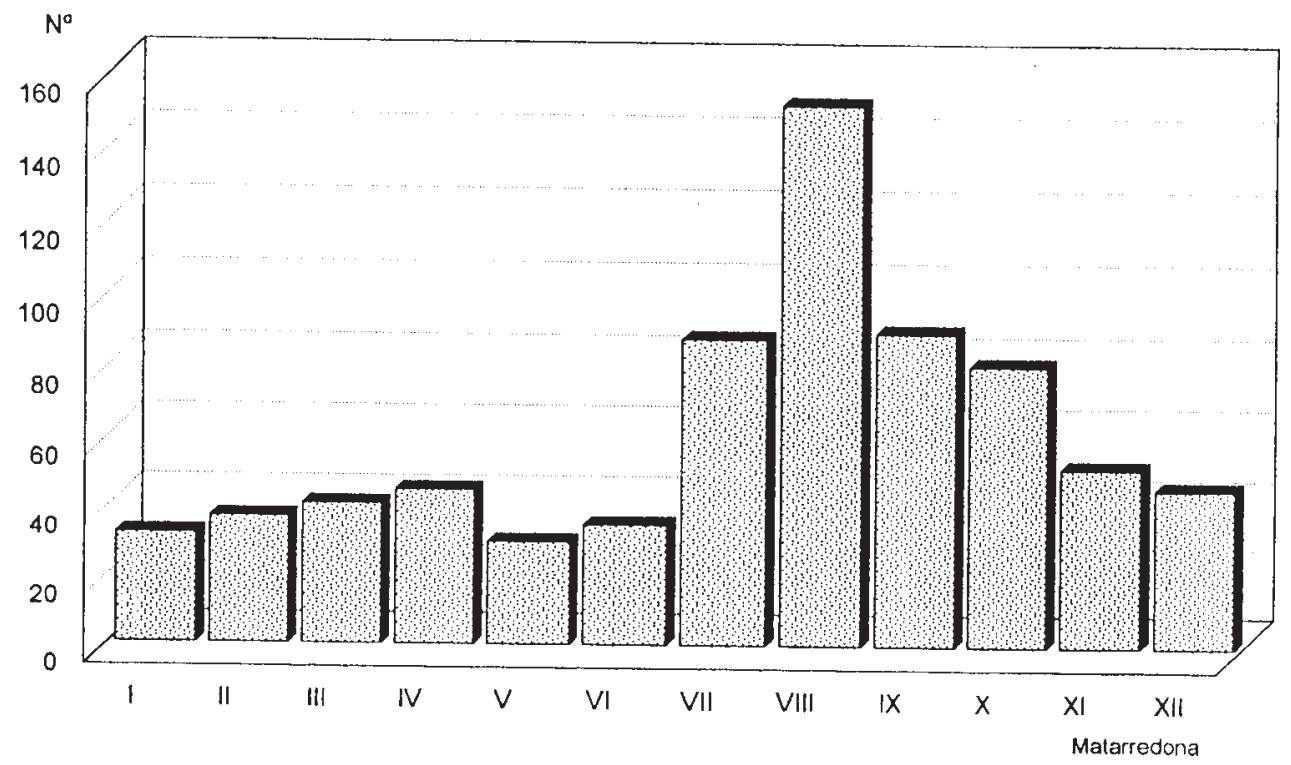

Figura 10. Condicionamientos ambientales y climáticos justifican la mayor frecuencia de los siniestros en el período junio-octubre, por lo que la superficie forestal afectada queda desprotegida frente a las lluvias de fuerte intensidad horaria de época otoñal, con el consiguiente riesgo de erosión.

En relación con la disminución de la capacidad de infiltración de los suelos, cobra mayor importancia la escorrentía superficial en las zonas afectadas por los incendios, toda vez que la capa de suelo fértil, que sustentaba la vegetación desaparecida, se pierde, facilitando así la actuación de los agentes erosivos; así, experimentan una notable aceleración los procesos de erosión provocados por el agua, que suponen el deterioro de las condiciones edáficas en las partes altas y el aterramiento de los embalses en las cotas inferiores, con el agravante de que, al producirse la mayoría de los incendios durante la época estival, las superficies forestales quedan desprotegidas frente a las lluvias de fuerte intensidad horaria características de los otoños (Figura 10).

En efecto, las pérdidas de suelo que suceden a la propagación de un siniestro forestal son verdaderamente graves (SANROQUE-RUBIO, 1981). Tras cada incendio las pérdidas de suelo son importantes; se podría estimar que después de cada fuego se pierde un centímetro de espesor edáfico, que necesitaría, como mínimo, de 200 a 300 años para volver a formarse. El fuego produce la combustión de la materia orgánica constitutiva de los horizontes edáficos superficiales, lo que lleva asociada una inmediata mineralización del mismo (PLATA-GUITIAN, 1966). El lavado posterior, a causa de las lluvias, hace que también este retraso se pierda por escorrentía y erosión. En consecuencia, además de quedar reducida la vegetación, sufren un enorme deterioro las condiciones de arraigo y de germinación de las plantas. La vegetación se ve obligada a desarrollar unos mecanismos de adaptación y de defensa frente a estas condiciones, de forma que se seleccionan las especies, al tiempo que logran sobrevivir al fuego.

Asimismo, la permanencia de árboles muertos en pie y de tocones predispone a la multiplicación de insectos y hongos xilófagos que constituirán una amenaza potencial para la masa vegetal aledaña. Pero, estos siniestros también comportan un cambio de las 
condiciones microclimáticas, con variaciones térmicas acusadas, menor infiltración, mayor evaporación e incremento de la acción desecadora del viento.

El fuego provoca también la reducción sensible de la población animal, especialmente ciertos grupos de invertebrados (gasterópodos, formícidos, arácnidos, etc.), y algunos vertebrados de pequeño tamaño (roedores, reptiles, etc.). Por otro lado, afecta al incremento de la virulencia de las avenidas fluviales producidas por precipitaciones de gran intensidad horaria, cuya escorrentía ya no tiene freno en su discurrir hacia los cauces; de tal modo que las laderas sometidas a incendios sufren graves pérdidas de espesor y calidad de suelos mediante procesos de erosión laminar o con la formación de regueros, precedentes, según las litofacies afectadas, de futuras cárcavas. Asimismo, la secuela del fuego se manifiesta con un paisaje en el que los esqueletos calcinados de los árboles quemados son los protagonistas.

\section{Conclusiones, propuestas y planes de actuación}

Ante esta situación sólo cabe concluir con una serie de propuestas que intenten dar solución al grave desastre ecológico y económico que estos sucesos provocan en el ámbito de La Montaña (Figura 11). Tales propuestas se sintetizan en:

* la necesidad de realizar un cambio en la política forestal, que pase por una revisión legislativa que tenga como objetivo adecuar la ley a las actuales circunstancias del monte, en especial a la valoración del patrimonio ecológico y a fomentar la defensa en los montes de propiedad privada.

* el uso, en las repoblaciones forestales, de especies autóctonas que generan menos maleza y cuya combustibilidad es menor. Del mismo modo, integrar la actividad repobladora con otras políticas sectoriales y considerar la importancia de la política hidrológica.

\section{Incendios forestales \\ La Montaña}

\section{SOLUCIONES}

Necesidad de realizar un cambio en la política forestal

Uso de especies autóctonas que generan menos maleza y son menos combustibles

Apertura de caminos que sirvan de cortafuegos

Mayor vigilancia

Sanciones más duras

Descentralización en la gestión de montes

Compromiso social que vincule a los ciudadanos con su medio ambiente

Procesos informativos y formativos que posibiliten adquisión conciencia social

Matarredona

FigURA 11 
* una mayor vigilancia y sanciones más duras para los culpables.

* la profesionalización de las brigadas de extinción, dotándolas de mejores equipos y una buena preparación técnica y física. En el área de vigilancia y extención debe aminorarse la velocidad de detención del fuego y aumentar la capacidad de transporte, que ha sido mejorada considerablemente en los últimos años mediante la utilización de helicópteros.

* unos procesos informativos y formativos que posibiliten la adquisición de una conciencia social sobre las consecuencias irreparables de los incendios forestales.

\section{Bibliografía}

ALTAMIRA y CREVEA, R. (1985): Derecho consuetudinario y economía popular en la provincia de Alicante, Ed. facsímil, Instituto Estudios «Juan Gil Albert», Alicante.

LIEBANA, P. y RIBARROCHA, V. (1981): El bosque y los incendios forestales, Diputación de Valencia.

MANSANET, C. Ma . (1987): Incendios forestales en Alicante. Estudio de la evolución de la vegetación quemada, Caja de Ahorros Provincial, Alicante, $188 \mathrm{pp}$.

MATARREDONA, E. (1996): «Atonía y agotamiento demográfico de La Montaña alicantina», Investigaciones Geográficas, $\mathrm{n}^{\circ}$ 15, Instituto Universitario de Geografía, Universidad de Alicante, pp. 41-60.

MONTIEL, C. (1990): Los montes de utilidad pública en la provincia de Alicante, Universidad de Alicante, $227 \mathrm{pp}$.

MUÑOZ, J. (1986): «Incendios forestales en la Comunidad Valenciana», El Campo, n 103, Banco de Bilbao, pp. 136-138.

NAVARRO CERRILLO, R. y XANDRI, P. (1995): «Repoblaciones forestales», Panda, Otoño 1995, pp. 8-11.

PLATA ASTRAY y GUITIAN OJEA (1966): «Transformaciones experimentadas en el suelo por la acción del fuego», Anales de Edafología y Agrobiología, CSIC, Madrid.

SANROQUE, P. y RUBIO, J.L. (1982): El suelo y los incendios forestales, Diputación Provincial, Valencia.

VÉLEZ, R. (1980): «Efectos del fuego y de la ordenación del combustible forestal en ecosistemas mediterráneos en España», Boletín de la Estación Central de Ecología, 17, ICONA, Madrid. 